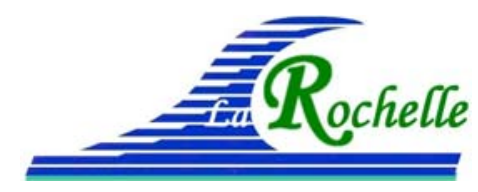

XVèmes Journées Nationales Génie Côtier - Génie Civil

La Rochelle, 29 au 31 mai 2018

DOI:10.5150/jngcgc.2018.079 ～(C) Editions Paralia CFL

disponible en ligne - http://www.paralia.fr - available online

\title{
Analyse économique de la gestion des plages : cas des banquettes de Posidonie sur les communes du littoral méditerranéen français
}

\author{
Alizée MARTIN ${ }^{1,2}$, Guillaume BERNARD ${ }^{2}$, Sylvaine IZE $^{2}$, Olivier HERLORY ${ }^{3}$
}

1. Direction de l'Environnement, de l'Aménagement et du Logement de la Martinique, Route de la Pointe de Jaham, BP7212, Schoelcher 97274, Martinique, France. alizee.martin@i-carre.net

2. Agence Française pour la Biodiversité, 26 rue de la République, 13001 Marseille, France.guillaume.bernard@afbiodiversite.fr; sylvaine.ize@afbiodiversite.fr

3. CREOCEAN, Agence PACA-CORSE, 230 avenue de Rome, Valparc-Bât B, 83500 La Seyne sur mer, France. herlory@creocean.fr

\section{Résumé :}

La posidonie (Posidonia oceanica) est une espèce protégée de plante à fleur sousmarine, endémique de la mer Méditerranée, qui constitue de vastes prairies entre la surface et $40 \mathrm{~m}$ de profondeur. Sur les plages de la région Provence Alpes Côte d'Azur, les amas de feuilles mortes de posidonies en bord de mer, appelés banquettes, sont à l'origine de conflits d'usage, obligeant les collectivités à trouver des modes de gestion permettant la préservation d'une espèce à haute valeur patrimoniale sans réduction de la valeur touristique de leurs plages.

En effet, les banquettes remplissent de nombreuses fonctions écologiques. Elles protègent contre l'érosion, en préservant la plage de l'assaut des vagues et en facilitant le piégeage de nouveaux arrivages de sable. Elles constituent un écosystème de type litière, source de matière et d'énergie pour le milieu côtier et l'arrière de plage via le réseau trophique détritique. Or ces amas de feuilles mortes peuvent être considérés comme un déchet, conduisant les collectivités littorales à les retirer. La posidonie étant une espèce protégée, la destruction de ses feuilles, même mortes, est interdite. C'est pourquoi différents modes de gestion des banquettes ont été développés par les communes pour concilier les attentes touristiques avec les enjeux environnementaux et économiques liés à la fréquentation des plages. Ainsi, les banquettes peuvent être laissées en place, stockées, déplacées sur une partie de plage, réimmergées, remaniées sous forme de "millefeuille", etc.

L'objet de cette étude est de réaliser une mise à jour de l'état des lieux des pratiques en matière de gestion des banquettes de posidonies et de préciser les coûts qui y sont liés. Dans ce cadre, des entretiens ont été menés avec 30 communes littorales de la région Provence Alpes Côte d'Azur. Ces échanges ont permis d'aborder les avantages et inconvénients de chaque mode de gestion. 


\section{Thème 6 - Gestion durable des zones littorales et estuariennes}

Au-delà des considérations écologique et directement économique, l'aspect touristique est un enjeu prédominant pour ces communes, directement impactées par l'attractivité et l'économie générées par leur littoral. Une meilleure compréhension de la formation des banquettes, de leur rôle contre l'érosion, de leur dégradation et un suivi scientifique permettant d'apporter les preuves de l'efficacité des modes de gestion seraient autant d'éléments à associer à une sensibilisation du public pour s'inscrire dans une démarche durable de gestion des banquettes de posidonies, et de manière plus générale de gestion du littoral.

\section{Mots-clés :}

Posidonia oceanica, Banquettes, Plages, Tourisme, Economie, Sensibilisation, Gestion.

\section{Introduction}

Les politiques de gestion des plages sont généralement élaborées en réponse aux exigences et aux attentes des usagers, afin de favoriser le développement de l'économie touristique. En régions Provence Alpes Côte d'Azur (PACA) et Corse, les laisses de mer constituées par les feuilles de posidonie (Posidonia oceanica) conduisent les collectivités littorales à chercher des modes de gestion conciliant la préservation des enjeux environnementaux liés à cette plante protégée tout en maintenant la valeur touristique des plages.

En effet, la posidonie est une plante marine endémique de Méditerranée, qui présente une haute valeur écologique et patrimoniale (VASSALLO et al., 2013). Elle constitue de vastes prairies entre la surface et $40 \mathrm{~m}$ de profondeur, dont les feuilles mortes, à l'automne, s'échouent sur les plages et s'accumulent sur plusieurs dizaines de centimètres de hauteur (figure 1) pour former des structures appelées banquettes (e.g. BOUDOURESQUE \& MEINESZ, 1982).

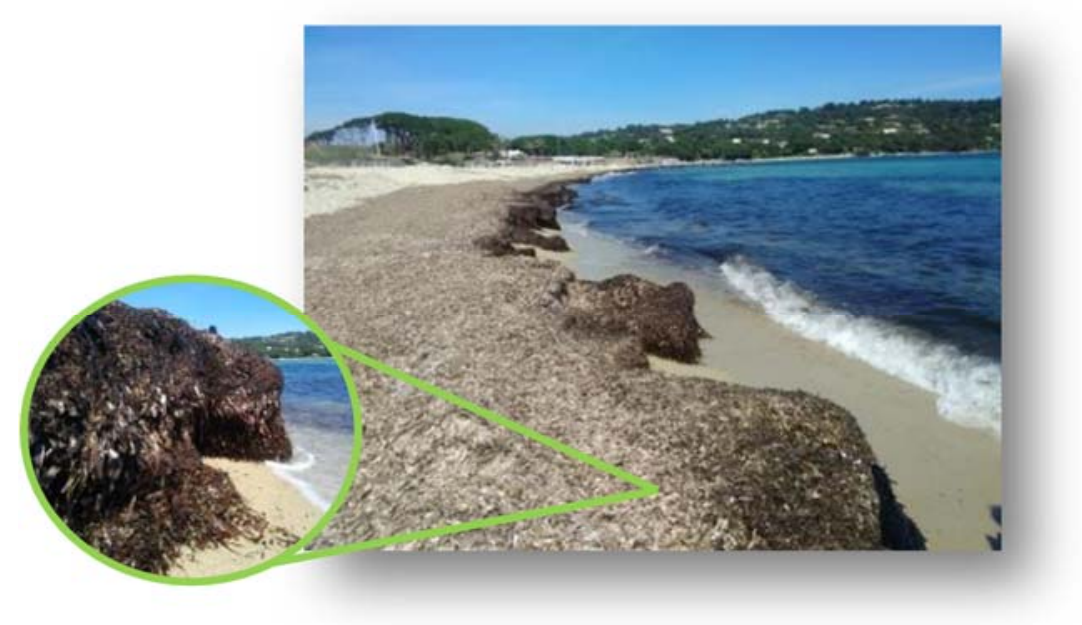

Figure 1. Photographie d'une banquette de Posidonie en bord de plage. 


\section{XVèmes Journées Nationales Génie Côtier - Génie Civil \\ La Rochelle, 29 au 31 mai 2018}

Ces formations peuvent être assimilées à une litière, source de matière et d'énergie pour le milieu côtier et l'arrière de plage via le réseau trophique détritique (e.g. MATEO et al., 2003). Elles constituent également des barrières naturelles de protection contre l'érosion (e.g. SIMEONE \& DE FALCO, 2012) et conséquemment limitent les coûts et les préjudices écologiques engendrés par les opérations de rechargement en sable. En dépit des enjeux induits par leur présence et de la réglementation sur les espèces protégées qui limite les actions sur les banquettes, elles sont généralement retirées pour répondre aux attentes esthétiques des estivants (CREOCEAN \& CSIL, 2011)

Avec l'idée à terme de proposer des solutions durables de gestion des banquettes de posidonie, cette étude a pour but de faire un état des lieux des pratiques des communes littorales et d'évaluer leur coût associé puis de les comparer sur la base d'une analyse multicritères intégrant les dimensions écologique, économique et touristique.

\section{Matériel et méthodes}

\subsection{Modes de gestion des banquettes de posidonie}

Cette étude s'appuie sur une série d'entretiens réalisés en 2017 auprès de 30 communes littorales sur les 57 que comptent la région PACA. Elle a permis de collecter des informations sur 142 plages correspondant à plus de $90 \mathrm{~km}$ de linéaire côtier.

Un questionnaire a été élaboré pour assurer une cohérence et une homogénéité dans la récolte des informations. Les entretiens semi-directifs, menés auprès des services techniques (commune, métropole ou SIVOM) en charge de la gestion des plages, avaient pour but d'aborder les questions liées :

1) à la gestion globale des plages (prestataire, engins, actions, ...) ;

2) au nettoyage des plages (moyens mobilisés, coûts, fréquence, ...) ;

3) à la gestion des banquettes de posidonies (présence, volume, actions menées, coûts, ...);

4) au rechargement des plages (matériaux utilisés, volume, coûts, ...) ;

5) à la sensibilisation du public en rapport avec les banquettes de posidonie (moyens, coûts, ...).

\subsection{Coûts liés à la gestion des banquettes de posidonie}

L'évaluation des coûts liés aux actions menées sur les banquettes de posidonie a été réalisée à partir des données fournies par les communes lors des entretiens. L'analyse a été affinée dans un second temps à partir des tarifs moyens de mobilisation des engins et des autres postes de dépenses afin de compléter les informations fournies par les gestionnaires. 


\section{Thème 6 - Gestion durable des zones littorales et estuariennes}

\subsection{Perception des banquettes de posidonie par les usagers}

Sans possibilité matérielle lors de cette étude d'évaluer la perception qu'ont les touristes des banquettes, cet aspect a été étudié à partir de l'exploitation des résultats des enquêtes menées par l'association Mer Terre à Six-Fours-les-plages en 2009 (MER TERRE, 2009) et l'association Méditerranée 2000 dans le cadre des stands Inf'eau Mer tenus sur les plages de PACA en 2016 (notamment à travers les réponses à la question "Pour vous, la présence de posidonie sur une plage signifie qu'elle est ?", INF’EAU MER, 2016).

\section{Résultats}

\subsection{Modes de gestion des banquettes de posidonie}

Les entretiens avec les communes ont permis de mettre en évidence 7 modes de gestion des banquettes de posidonie différents (figure 2). Ces pratiques dépendent principalement de la vocation des plages (aménagements, accessibilité), de leur configuration et de la sensibilité écologique des élus.

1) Laissées en place ( $18 \%$ des cas) : les banquettes sont laissées sur la plage, sans aucune intervention ou remaniement. Elles peuvent repartir seules à la mer ou être dispersées par le vent. Une couche de banquette peut persister durant la saison estivale.

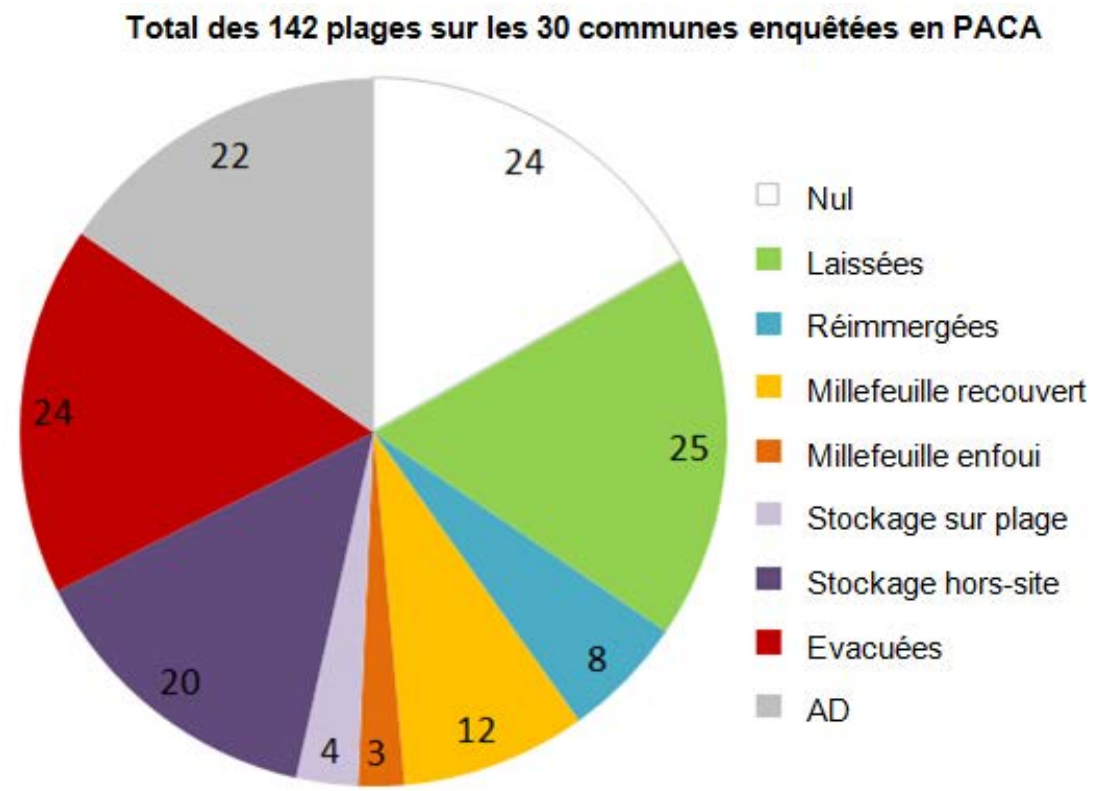

Figure 2. Modes de gestion des banquettes de posidonie sur les 142 plages des 30 communes enquêtées en PACA en 2017. La catégorie "Nul" correspond aux plages qui ne présentent pas de banquettes. La catégorie "AD" représente les plages pour lesquelles la donnée est manquante. 


\section{XVèmes Journées Nationales Génie Côtier - Génie Civil \\ La Rochelle, 29 au 31 mai 2018}

2) Réimmergées (6\% des cas) : les banquettes, après avoir été laissées en place une partie de l'année, sont repoussées en mer. L'intervention s'effectue lorsque les conditions météorologiques (vent et courant) sont favorables à leur dispersion loin de la côte.

3) Millefeuille recouvert ( $8 \%$ des cas) : les banquettes, après avoir été laissées en place une partie de l'année, sont étalées sur la plage. Les amas de feuilles mortes de posidonie sont répartis sur toute la surface de la plage puis recouverts de sable. Ce sable peut être prélevé sur la plage ou être importé. Les banquettes restent ainsi sur leur plage d'origine mais sont cachées sous une épaisseur de sable plus ou moins importante.

4) Millefeuille enfoui ( $2 \%$ des cas) : les banquettes, après avoir été laissées en place une partie de l'année, sont enfouies sous la plage. Des tranchées de longueur variable sont creusées, souvent éloignées du bord de l'eau. Les feuilles mortes de posidonie sont prélevées puis déposées au fond des tranchées en alternance avec des couches successives de sable.

5) Stockage sur la plage (3\% des cas) : les banquettes sont prélevées en début de saison touristique puis déplacées. Les feuilles mortes de posidonie peuvent être regroupées en tas dans une zone restreinte, ou étalées dans des zones moins fréquentées ou encore sujettes à l'érosion. Après la saison touristique, les amas de feuilles mortes de posidonie peuvent être laissés sur leur lieu de stockage, ré-étalés sur la plage ou encore repoussés à la mer.

6) Stockage hors-site (14\% des cas) : les banquettes sont prélevées sur la plage en début de saison touristique puis déplacées en dehors de la plage. Elles sont entreposées sur un terrain dédié pendant la saison touristique puis remises sur les plages en hiver.

7) Evacuées (17\% des cas) : les banquettes sont prélevées sur la plage puis évacuées. Elles sont parfois envoyées en déchèterie (en tant que déchets verts ou ordures ménagères). Ces deux finalités ont été regroupées dans une même catégorie puisqu'il s'agit dans les deux cas de pratiques interdites par la loi, constituant un retrait total des banquettes de leur milieu d'origine.

\subsection{Coûts liés à la gestion des banquettes de posidonie}

L'estimation des coûts liés à chaque mode de gestion des banquettes de posidonie a permis de mettre en évidence que le fait de laisser les banquettes en place est la méthode la moins onéreuse (tableau 1). Cependant, cette estimation ne prend pas en compte la potentielle nécessité d'entretien des banquettes (création d'accès, nettoyage manuel des macrodéchets, ...), la sensibilisation du public à la présence de ces amas de feuilles mortes.

La ré-immersion des banquettes de posidonie nécessite la mobilisation d'un engin pour repousser les amas de feuilles mortes dans l'eau, soit un coût total d'environ $0,9 € / \mathrm{m} 3$ de banquette (tableau 1). En fonction des échouages, l'opération de ré-immersion peut 


\section{Thème 6 - Gestion durable des zones littorales et estuariennes}

être renouvelée plusieurs fois dans la saison, ce qui peut multiplier le coût de mise en œuvre.

Le remaniement des banquettes sous forme de millefeuilles (qu'ils soient enfouis ou recouverts) mobilise au moins deux engins et peut nécessiter un rechargement en sable. Le coût de mise en œuvre de cette technique varie entre $19,9 € / \mathrm{m} 3$ pour un millefeuille enfoui et $89,9 € / \mathrm{m} 3$ pour un millefeuille recouvert, nécessitant un rechargement en sable (tableau 1).

Le stockage des banquettes sur la plage ou hors-site est une opération qui mobilise également plusieurs engins. Cette pratique implique une intervention en fin de saison estivale afin de ramener les feuilles mortes sur la plage. Les frais de transport sont la principale source de variation des coûts de mise en œuvre, qui varient entre 18,4 et $39,3 € / \mathrm{m} 3$ de banquette (tableau 1 ).

L'évacuation des banquettes apparaît comme étant le mode de gestion le plus coûteux (entre 83,4 et $168,4 € / \mathrm{m} 3$ de banquette), les frais de mise en décharge ayant un poids prépondérant dans cette estimation (tableau 1). Cette pratique est interdite par loi et elle expose le contrevenant à une amende $15000 €$ pour atteinte à la conservation d'espèces végétales non cultivées et/ou d'habitats naturels selon 1'article L.415-3 du Code de l'environnement.

Tableau 1. Estimation du coût par $\mathrm{m}^{3}$ de banquette de posidonie des modes de gestion identifiés.

Mode de gestion

Laissées en place

Réimmergées

Millefeuille recouvert ou enfoui

Stockage sur la plage ou hors-site

Evacuées
Coût moyen

( $€ / \mathrm{m}^{3}$ de banquette de posidonie)
0,9

19,9 à 89,9

18,4 à 39,3

83,4 à 168,4

\subsection{Perception des banquettes de posidonie par les usagers}

Les résultats de l'enquête du réseau Inf'eau Mer de 2016 (INF'EAU MER, 2016) ont mis en évidence que la grande majorité des personnes interrogées (71\% des 4133 questionnaires récoltés) ne voit pas la posidonie comme une nuisance, la considérant comme un élément naturel de la plage. Seule une faible proportion de gens (5\%) juge une plage sale à la présence de feuilles mortes de posidonie (figure 3). 


\section{$X V^{\text {èmes }}$ Journées Nationales Génie Côtier - Génie Civil \\ La Rochelle, 29 au 31 mai 2018}

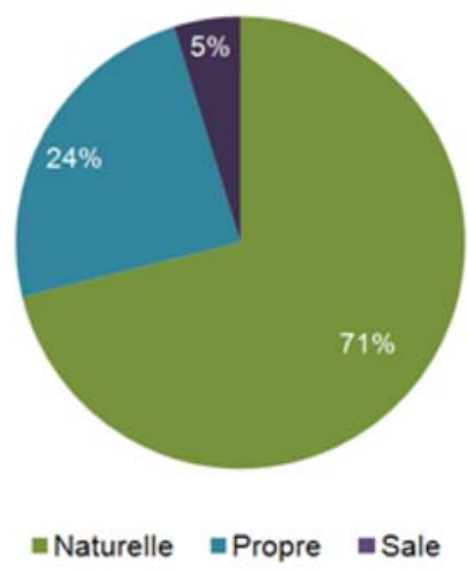

Figure 3. Répartition des réponses à la question "Pour vous, la présence de posidonie sur une plage signifie qu'elle est ?", exprimée en \% (d'après INF'EAU MER 2016).

Les résultats de l'étude réalisée par Mer Terre sur la commune de Six-fours en 2009 (MER TERRE, 2009) vont dans le même sens, montrant que la majorité des estivants (72\% des 251 personnes enquêtées) n'est pas incommodée par les banquettes de posidonie soit parce ce que leur présence ne les dérange pas ou parce qu'ils estiment qu'il est naturel et écologique qu'il y ait des feuilles sur les plages et sont satisfaits que les pouvoirs publics en tiennent compte.

\section{Conclusions}

Les différents modes de gestion des banquettes de posidonie pratiqués en région PACA en 2017 ont été comparés par une analyse multicritères afin d'évaluer leur durabilité sur le plan environnemental, économique et touristique (tableau 2).

L'analyse comparative atteste de l'intérêt majeur sur le plan écologique et économique de laisser les banquettes sur les plages (tableau 2). Le principal point de blocage de cette pratique réside dans l'idée que se font les gestionnaires de la perception des banquettes par les estivants alors que les enquêtes tendent à montrer qu'il y a une acceptation de ces formations en tant qu'élément naturel des plages pour la majorité des touristes (INF'EAU MER, 2016, MER TERRE, 2009). Cette différence de perception souligne la nécessité d'investiguer les services écosystémiques rendus par les banquettes de posidonie, point sur lequel les données sont encore manquantes (VASSALLO et al., 2013). 


\section{Thème 6 - Gestion durable des zones littorales et estuariennes}

Tableau 2. Comparaison multicritères des modes de gestion des banquettes de posidonie établie suite aux entretiens avec les 30 communes de PACA enquêtées en 2017. Le code couleur permet de faire ressortir les bénéfices (en vert) et inconvénients (en rouge) de chaque mode de gestion.
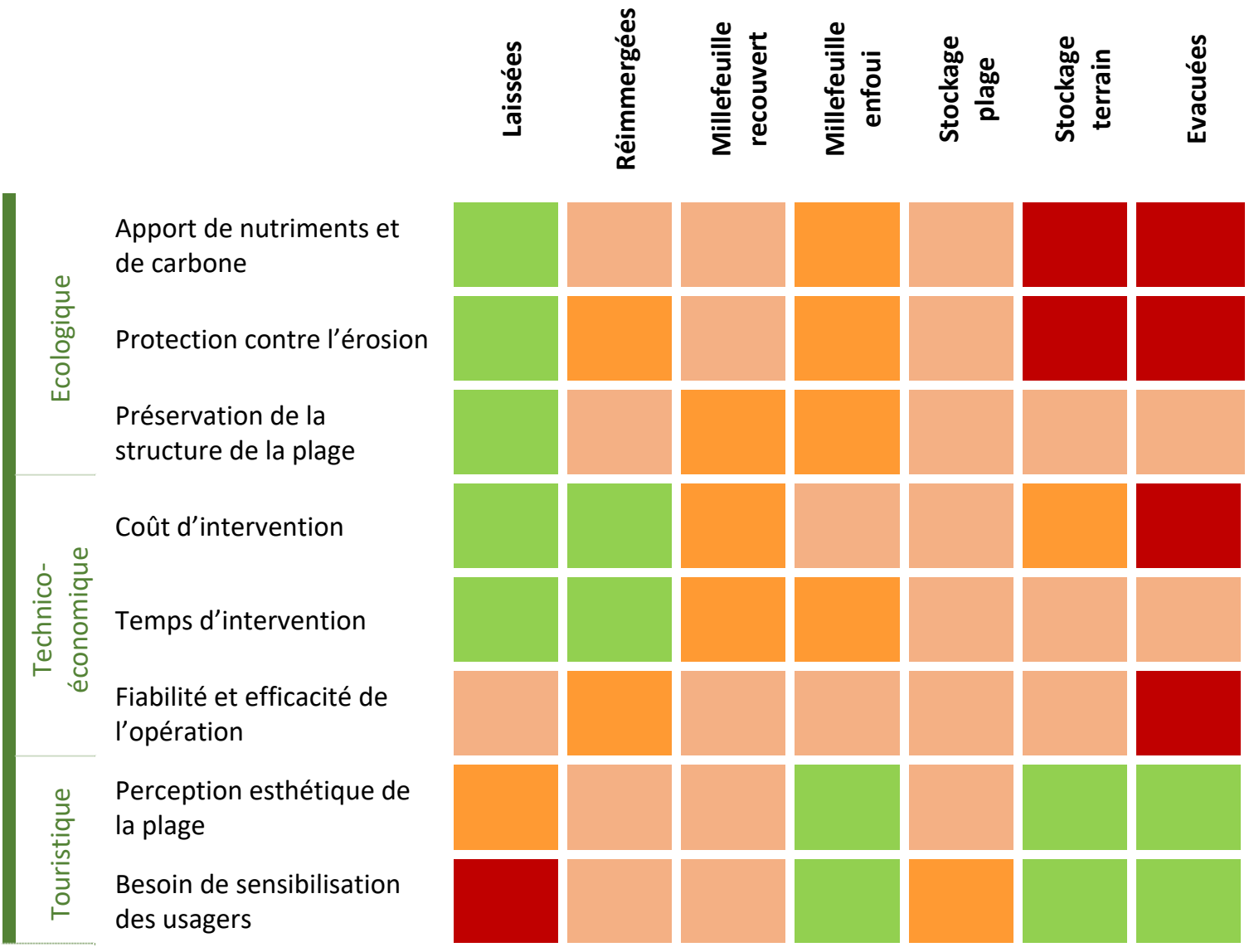

Les modes de gestion alternatifs comme la réimmersion, le millefeuille ou le stockage des feuilles mortes de posidonie sur la plage sont autant de méthodes qui constituent des solutions douces sur le plan écologique et économique (tableau 2). Cependant l'évaluation de leur rôle écologique reste encore parcellaire et nécessiterait d'être approfondie.

L'évacuation est le mode de gestion présentant le plus d'inconvénient sur le plan économique et écologique (tableau 2), d'autant qu'elle est illégale puisqu'elle suppose la destruction d'une espèce protégée.

Cette étude a mis en évidence que les modes de gestion des banquettes de posidonie étaient basées sur des pratiques empiriques contraintes par la réglementation sur les espèces protégées. Pour répondre aux attentes des gestionnaires, l'idéal serait de pouvoir apporter une base scientifique à ces méthodes afin d'aller vers la mise au point une d'une solution intégrée de gestion durable en permettant de préserver leur rôle 


\section{XVèmes Journées Nationales Génie Côtier - Génie Civil La Rochelle, 29 au 31 mai 2018}

structurel en tant que barrière de protection contre l'érosion et leur rôle fonctionnel en tant que source de carbone et de nutriments sans réduire la valeur touristique des plages.

\section{Références bibliographiques}

BOUDOURESQUE C.F., MEINESZ A. (1982). Découverte de l'herbier de Posidonie. Cah. du Parc Natl. Port Cros, Vol. 4, pp 1-79. URL :http://mio.pytheas.univ-amu.fr/gisposidonie/wpcontent/uploads/2015/02/Boudouresque_Meinesz.pdf

CREOCEAN, CSIL. (2011). Bilan de la gestion des banquettes de posidonie en Région Provence-Alpes-Côte-d'Azur. Rapport. DREAL PACA, ADEME, Région PACA. URL : http://csil.free.fr/BilanGestionBanquettesPosidoniePACA20102011.pdf

INF'EAU MER. (2016). Bilan de l'enquête d'opinion 2016, Régions Provence-AlpesCôte d'Azur et Corse. Rapport.

MATEO M., SANCHEZ-LIZASO J., ROMERO J. (2003). Posidonia oceanica "banquettes": a preliminary assessment of the relevance for meadow carbon and nutrients budget. Estuarine, Coast Shelf, Vol. 56, pp 85-90. https://doi.org/10.1016/S02727714(02)00123-3

MER TERRE (2009). Programme de gestion raisonnée des plages de la commune de Six-fours. Rapport. Commune de Six-Fours-Les-Plages, Dir. Générale des Serv. Coord. des actions liées au Développement Durable.

SIMEONE S., DE FALCO G. (2012). Morphology and composition of beach-cast Posidonia oceanica litter on beaches with different exposures. Geomorphology, Vol. 151-152, pp 224-233. https://doi.org/10.1016/j.geomorph.2012.02.005

VASSAllO P., PAOLI C., ROVERE A., MONTEFALCONE M., MORRI C., BIANCHI C. N. (2013). The value of the seagrass Posidonia oceanica: A natural capital assessment. Mar. Pollut. Bull., Vol. 75(1-2), pp 157-167. https://doi.org/10.1016/j.marpolbul.2013.07.044 
Thème 6 - Gestion durable des zones littorales et estuariennes 